discharge of sulphur and nitrogen ${ }^{4}$. During subsequent work on the behaviour of the above products of the nitrous oxide-hydrogen interaction, reversal of change and also a full periodic effect as in the accompanying figure are observed under certain conditions of the decomposition of nitrogen dioxide by the silent discharge ${ }^{4}$.

S. S. Joshi.

G. S. Deshmukh.

Chemical Laboratories,

Benares Hindu University. Dec. 21.

1 Joshi, Trans. Faraday Soc., (a), 25, 139 (1929); (b) 25, 129 (1927); Curr. Sci., 8, 548 (1939); 13, 153 (1944); Nature, 154, 147 (1944). ${ }^{2}$ Hinshelwood, Proc. Roy. Soc., A, 108, 292 (1924). Hutchison and Hinshelwood, J. Chem. Soc., 129, 1556 (1926). Melville, Proc. Roy. Soc., A, 142, 524 (1933). Dixon, J. Amer. Chem. Soc., 57, 818 (1935). Cassel and Glückauf, $Z$. phys. Chem., B, 19, 47 (1932) ${ }^{3}$ Hedges and Myers, "Physico-Chemical Periodicity" (London, 1926). 4 Joshi, Pres. Address, Chem. Sec., Indian Sci. Cong. (1943).

\section{Compounds of the Structural Type of Calcium Titanate}

IN a previous communication ${ }^{1}$, it was recorded that precision X-ray powder mothods with a largediameter camera show that the structure of nickel oxide is not strictly cubic, but only pseudo-cubic. Splitting of some of the X-ray reflexions into more than one component is explained if a small distortion of the cubic lattice along an octahedral axis is assumed, so that the true symmetry is rhombohedral hexagonal.

In the course of investigations on compounds of the structural type of perovskite, $\mathrm{CaTiO}_{3}$, it has been found that precision powder methods are capable of demonstrating that only a minority of these crystals have cubic symmetry. Naray-Szabo ${ }^{2,3}$ has recently examined many crystals of the calcium titanate type by means of oscillation diagrams, and has shown that several, including perovskite itself, are monoclinic, and others, for example, lead titanate, are tetragonal or orthorhombic. Using the powdered crystal technique, the differentiations described by Naray-Szabo have been, in general, confirmed, although a small distortion from cubic symmetry has been observed for barium titanate, which is still classed as cubic by Naray-Szabo.

The X-ray powder photographs may be grouped into three main types. For compounds typified by strontium titanate the lines fall in the sequence demanded by the ideal cubic structure. No extra reflexions occur and no splitting of the main reflexions can be distinguishod. The ideal cubic structure is therefore valid to a high degree of accuracy.

For some other compounds, of which barium titanate is an example, many of the powder lines are split into more than one component, and the new features can be explained if the symmetry is assumed to be tetragonal. The measured lattice constants of barium titanate are then $a_{0}=3.9866 \mathrm{kX}$., $c_{0}=$ $4.0195 \mathrm{kX}$. at $22^{\circ} \mathrm{C}$. For lead titanate a still greater distortion of the structure is revealed, the axial ratio being much further removed from unity.

With a larger group of compounds the main powder lines show many complexities and a number of extra reflexions appear in intermediate positions. Part of a powder photograph of calcium stannate is reproduced herewith to indicate the kind of effect which is observed. The main groups of lines have been indexed according to the old cubic structure. One noteworthy

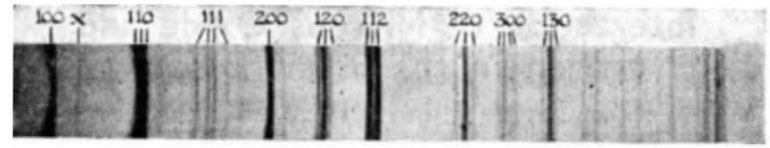

PART OF X-RAY POWDER PHOTOGRAPH (COPPER Ka RADIATION, 19 CM. CAMERA) OF CALCIUM STANNATE (CaSnO

and characteristic feature of the X-ray pattern of crystals of this type is that a low-order line $(x)$ appears the spacing of which is $2 / \sqrt{ } 5$ that of 100. This indicates that the indices of the main lines should in fact be doubled, and therefore, when an approximation to cubic symmetry is assumed, the unit cell dimensions must be twice those of the ideal perovskite unit cell. For example, the side of the pseudo-cubic unit cell of calcium stannate is approximately $7.87 \mathrm{kX}$., and not $3.935 \mathrm{kX}$., the lattice constant for the old structure.

It will be seen that all the members of this large class of compounds, having the general formula $A B \mathrm{O}_{3}$, cannot be regarded as isomorphous in the strict sense of the term. Although the ideal lattice has cubic symmetry, in the majority of cases, the cubic structure is appreciably distorted and the true symmetry may be tetragonal or more often monoclinic. There are, no doubt, other groups of compounds, the members of which have similar structural relationships, which would not be embraced by the term isomorphism as at present understood. Naray$\mathrm{Szabo}^{3}$ suggests that such related structures should be described as 'sister' structures. An alternative would be to use the term 'pseudo-isomorphous' to denote the close structural similarities. This would have the advantage that it follows the accepted custom of using the prefix 'pseudo' to denote structures that are distorted but closely related to the more symmetric crystal systems.

Research Laboratories,

H. P. Rooksby.

General Electric Company, Limited, Wembley.

Rooksby, H. P., Nature, 152, 304 (1943).

2 Naray-Szabo, I., Naturwiss., 31, 202 (1943).

Naray-Szabo, I., Naturwiss., 31, 466 (1943).

\section{Crystal Structure of Barium Titanate}

IT is well known that barium titanate belongs to the group of compounds having structures of the perovskite type ${ }^{1}$. The ideal perovskite structure (G5 in the "Strukturbericht") has a simple cubic lattice, with one formula-weight per cell, the atomic parameters being as follows : 2 -valent cation, $(0,0,0)$; 4 -valent cation, $\left(\frac{1}{2}, \frac{1}{2}, \frac{1}{2}\right)$; oxygens, $\left(0, \frac{1}{2}, \frac{1}{2}\right),\left(\frac{1}{2}, 0, \frac{1}{2}\right)$, $\left(\frac{1}{2}, \frac{1}{2}, 0\right)$. It was early recognized ${ }^{2}$ that for many of these compounds, including perovskite $\left(\mathrm{CaTiO}_{3}\right)$ itself as well as barium titanate, the structure was not truly cubic, but was actually a slightly deformed modification of it. Perovskite itself is generally believed monoclinic; the structure has recently been determined in detail by Naray-Szabo ${ }^{3}$, who finds a monoclinic unit cell with all its edges doubled relative to the unit cell of the ideal structure. No detailed work on barium titanate has hitherto been published, and it was thought of interest to investigate it. Powder photographs of the synthetic material taken in a $19 \mathrm{~cm}$.-diameter camera with copper $K \propto$ radiation provided the data for determining the structure.

The structure is tetragonal, the dimensions of the unit cell at $20^{\circ} \mathrm{C}$., for a typical sample of material, 